\title{
Development of a high-performance immunolatex based on "soft landing" antibody immobilization mechanism
}

\author{
Xiaofei Yuan ${ }^{1,2,3}$, Dolça Fabregat ${ }^{5}$, Keitaro Yoshimoto $^{2}$ and Yukio Nagasaki Y,2,3** $^{\text {(2) }}$ \\ ${ }^{1}$ Department of Materials Sciences, Graduate School of Pure and Applied Sciences, University \\ of Tsukuba; ${ }^{2}$ Tsukuba Advanced Research Alliance (TARA), and ${ }^{3}$ Master School of Medical \\ Sciences, Graduate School of Comprehensive Human Sciences, University of Tsukuba; \\ ${ }^{4}$ Satellite Laboratory of International Center for Materials Nanoarchitectonics (MANA) in \\ National Institute for Materials Science (NIMS), University of Tsukuba, Tennodai 1-1-1, \\ Tsukuba, Ibaraki 305-8573, Japan; and ${ }^{5}$ Biokit Latex R\&D Department, \\ 08186 Lliçà d'Amunt, Barcelona, Spain.
}

"To whom correspondence should be addressed.

Tel: +81-29-853-5749; Fax: +81-29-853-5749; E-mail:yukio@nagalabo.jp

Abstract: Rabbit anti-human ferritin (anti-hFT) polyclonal immunoglobulin G (IgG) and poly(ethylene glycol) (PEG) were sequentially co-immobilized onto polystyrene submicroparticles (sMPs) to construct sMP/anti-hFT/PEG (SAP) immunolatex. Chemical immobilization of anti-hFT was performed at different $\mathrm{pH}$ levels to evaluate variations in antigen recognition. Basic $\mathrm{pH}$ disfavored conjugation of anti-hFT to sMPs, but remarkably increased its antigen recognition in comparison to that at neutral $\mathrm{pH}$. We investigated this 
intriguing phenomenon further by assessing the kinetics of antibody binding, including the time-dependency of immobilization, antigen recognition, and orientation of bound anti-hFT. Therefore, we attributed high antigen recognition to significant electrostatic repulsion between sMPs and anti-hFT at basic $\mathrm{pH}$, which predominately prevented anti-hFT access to sMPs and concurrently promoted anti-hFT orientations suitable for antigen recognition. Subsequent PEG modification maintained such anti-hFT orientation, without which antigen-accessible orientations would have decreased with time. Thus, properly oriented antibody and immediate PEGylation after antibody immobilization contributed to the formation of a high-performance SAP immunolatex.

Keywords: Antibody immobilization, Antibody orientation, PEGylation, Immunolatex, Immunoassay, Immunoreactivity

\section{Introduction}

With the development of biotechnology and nanotechnology, immobilized proteins, especially antibodies and enzymes, have been widely used as biosensors [1], microarrays [2-5], and immunoparticles [6-8]. These applications require immobilized proteins to maintain their conformation and proper active site orientation toward the bulk solution. Protein immobilization, however, is a complicated process, mainly owing to the unique structural features of proteins, such as diversity, flexibility, and amphiphilic surface, thereby making the process difficult to control. 
Antibody immobilization consists of physical adsorption and chemical binding, which depends on the driving force. The former is simple, but nonspecific attractive forces easily cause antibody desorption [9]. Chemical binding was proposed as a solution because stable covalent linkages with antibodies are formed. However, it likely results in random antibody conjugation, accompanied by reduced antigen recognition and/or denaturation [10,11]. Accordingly, controllable covalent binding with proper antibody orientation is crucial for desirable antibody immobilization. To date, much effort has been devoted to this challenging task. Several traditional methods have been developed, such as disulfide bonds at the constant moiety (Fc region) of IgG antibodies [12,13], site-specific modification of carbohydrates in the Fc region [14], acid pretreatment promoting the preferential Fc adsorption [15-17], and attachment via Fc-recognizing protein A [18] or protein G [19]. Use of these methods has resulted in some success in manipulating antibody orientation for efficient antibody-antigen interactions. From the viewpoint of industrial applications, however, all these strategies are still economically unfeasible because of complexity and length, coupled with the laborious procedures and special techniques involved in these strategies.

Versatile driving forces work during antibody physisorption, dependending on the features of antibodies and substrates [20-23], and the electrostatic interaction between them is one of the most important forces. Previous studies have shown that both the amount of immobilized antibodies and their orientation are affected by electrostatic interactions [21,22,24,25]. 
Simulation of antibody adsorption and orientation on a charged surface was also performed to support this kind of orientation control [23]. Even with chemical binding, the orientation of an antibody may be dramatically influenced by its physisorption properties. These properties are dependent on the substrate, because antibodies generally need to undergo physisorption prior to covalent conjugation [26]. We predict that controlling the interaction between antibodies and the substrate surface, by charging character of solid surface with proper $\mathrm{pH}$, ionic strength, etc., may easily adjust the orientation of the antibody during chemical binding. Thus, combining physical orientation control with robust chemical linkage between antibodies and substrates is an efficient strategy for desirable antibody immobilization.

In this study, rabbit anti-human ferritin (anti-hFT) polyclonal immunoglobulin $\mathrm{G}$ (IgG) was chemically co-immobilized with poly(ethylene glycol) (PEG) onto polystyrene submicroparticles (sMPs) for development of sMP/anti-hFT/PEG (SAP) immunolatex. (Scheme 1) To optimize its performance, the effects of immobilization conditions on anti-hFT were evaluated in detail with respect to the degree of immobilization, antigen recognition, and orientation. Electrostatic repulsion between anti-hFT and sMPs at basic $\mathrm{pH}$ inhibited anti-hFT access to the sMP surface, but promoted its antigen-accessible orientation on the surface, resulting in high antigen recognition capacity of immobilized anti-hFT (named "soft landing" mechanism). Both the "soft landing" mechanism and subsequent PEGylation, which maintain the co-immobilized antibody's orientation, are indispensable for constructing a 
high-performance SAP immunolatex.

\section{Materials and methods}

2.1 Materials. Aqueous suspension of carboxylated polystyrene sMPs $(10 \% \mathrm{w} / \mathrm{v}$, without surfactant) $[27,28]$, bovine serum albumin (BSA), rabbit anti-human ferritin (anti-hFT) polyclonal $\mathrm{IgG}$ ( $9.76 \mathrm{mg} / \mathrm{mL}, \mathrm{pH} 7.0$, isoelectric point $(\mathrm{pI})=6 \sim 7)$, human ferritin $(\mathrm{hFT})$ from spleen, and human sera from patients with rheumatoid arthritis (containing $29 \mathrm{ng} / \mathrm{mL}$ rheumatoid factor (RF); lot XI1005) and from healthy persons (without RF) were a kind gift from Biokit S.A. (Barcelona, Spain). According to the manufacturer's data, the surface charge density of the sMPs is $25.67 \mu \mathrm{C} \mathrm{cm}^{-2}$, and the area occupied by each carboxyl functional group is $62.4 \AA^{2}$. The particle size $(251 \mathrm{~nm})$ and electrophoretic mobility $\left(\mu_{\mathrm{e}}=-4.45\right.$ $\mu \mathrm{mcm} / \mathrm{Vs})$ of the sMPs in borate buffer solution $(10 \mathrm{mM}, \mathrm{pH} 8)$ were measured on a Malvern

Zetasizer Nano ZS instrument (He-Ne laser, U.K.). $\alpha$-Methoxy-poly(ethylene glycol)-pentaethylenehexamine (mPEG-N6, $M_{n}=6000 \mathrm{~g} / \mathrm{mol}$; Scheme 1a) $[27,28]$ aqueous solution ( $2 \% \mathrm{w} / \mathrm{v}, \mathrm{pH} 10)$ was provided by JSR Co. (Tokyo, Japan). Unless otherwise stated, mPEG-N6 was always diluted in phosphate buffer (PB, $\mathrm{pH} 7.4)$ and $0.01 \mathrm{M} \mathrm{HCl}$ to achieve a final concentration of $0.3 \% \quad \mathrm{w} / \mathrm{v} \quad\left(\begin{array}{llllll}8 & \mathrm{mM} & \mathrm{PB}, & \mathrm{pH} & 7.4) \text {. }\end{array}\right.$ 1-Ethyl-3-[3-dimethylaminopropyl]carbodiimide hydrochloride (EDC, special grade) and 2-morpholinoethanesulfonic acid (MES) were provided by Kanto Chemical Co., Inc. (Tokyo, Japan) and Dojindo Laboratories (Kumamoto, Japan), respectively. All buffers used in this 
study were prepared from sodium salts, deionized water (Millipore Milli-Q), and $1 \mathrm{M} \mathrm{NaOH}$ aqueous solution for $\mathrm{pH}$ adjustment. Unless otherwise stated, all experiments were performed at $25^{\circ} \mathrm{C}$ or room temperature.

\subsection{Methods}

\subsubsection{Antibody immobilization at different $\mathrm{pH}$ levels and salt concentrations.}

To estimate the effect of ionic strength by salt addition, low-concentration buffer solutions (ionic strength, $I=0.6 \mathrm{mM}$ ) were used in this experiment. As previously reported [27], a stoichiometric amount of EDC aqueous solution $(3 \mu \mathrm{L}, 12.5 \mathrm{mg} / \mathrm{mL}, \mathrm{EDC} / \mathrm{COOH}$ of sMPs $=$ 1) was added to a mixture of and sMP suspension (30 $\mu \mathrm{L})$ and MES buffer $(167 \mu \mathrm{L}, 3 \mathrm{mM}$, $\mathrm{pH}$ 5.5) to activate sMP carboxyl groups. The mixture was then shaken for $20 \mathrm{~min}$. The EDC-activated sMPs (30 $\mu \mathrm{L}$, with active ester groups on the surface) were further diluted with $263 \mu \mathrm{L}$ of $\mathrm{PB}(\mathrm{pH} 7.4, \mathrm{I}=0.6 \mathrm{mM}$, containing $0-18 \mathrm{mM} \mathrm{NaCl})$ or borate buffer $(\mathrm{pH}$ 8.0-9.5 with the same $\mathrm{I}$ and $\mathrm{NaCl}$ concentration) to alter the $\mathrm{pH}$ and/or salt concentration. Then, freshly diluted anti-hFT in corresponding buffer $(9 \mu \mathrm{L}, 1 \mathrm{mg} / \mathrm{mL})$ was fed and incubated for $1 \mathrm{~h}$ to allow the immobilization reaction between amine residues and the sMP active ester groups to occur. Low $\mathrm{pH}(<7.4)$ was unsuitable for this study.

After anti-hFT immobilization, the sMP/anti-hFT conjugates (sensitized sMPs) were separated by centrifugation $\left(15,000 \mathrm{rpm}\right.$ for $20 \mathrm{~min}$ at $25^{\circ} \mathrm{C}$; KR-20000; KUBOTA Co., Tokyo, Japan). A Micro BCA protein assay kit (\#23235; Thermo Fisher Scientific Int., IL) 
was used to quantify both sMP-bound and free anti-hFT, as previously reported [27].

To measure the reactivity of bound anti-hFT with a high signal-to-noise ratio and to colloidally stabilize the sensitized sMPs for various experiments, the residual bare sMP surfaces after antibody binding were further covered with mPEG-N6 for 30 min to form the sMP/anti-hFT/PEG immunolatex (SAP) through the covalent reaction between multiple mPEG-N6 amine end groups $(0.3 \% \mathrm{w} / \mathrm{v}$ in $0.27 \mathrm{mM} \mathrm{PB}, \mathrm{pH} 7.4)$ and the active ester groups on the surface. In this treatment, the final sMP and mPEG-N6 concentrations were both $0.1 \%$ w/v. The SAP immunolatex preparation protocol is illustrated in Scheme 1b. The resulting SAP immunolatex was centrifuged at $60,000 \mathrm{rpm}$ for $20 \mathrm{~min}$ at $25^{\circ} \mathrm{C}$ (CS $150 \mathrm{GX}$; Hitachi Koki Co. Ltd., Japan) to eliminate unbound antibody in the supernatant, which would otherwise competitively recognize antigens during the reactivity assay. The collected purified SAP immunolatex was homogeneously resuspended in borate buffer $(\mathrm{pH} 8.0,10 \mathrm{mM}$, containing $0.9 \mathrm{~g} / \mathrm{L} \mathrm{NaN}_{3}$ ) by ultrasonication $(35 \mathrm{~W}, 60 \mathrm{~Hz}, \sim 20 \mathrm{~s}$ for several rounds), followed by storage at $4^{\circ} \mathrm{C}$ for 1 day before use. Note that all the SAP immunolatex samples prepared at different $\mathrm{pH}$ levels were monodispersed and similar in size (261-268 nm). We did not observe any variations in these properties before and after redispersion prior to reactivity measurements.

On the basis of the results of these experiments, buffer concentrations were optimized and used in the following experiments to enhance buffering capability without promoting 
aggregation of sensitized sMPs.

\subsubsection{Effects of antibody-binding time.}

EDC-activated sMPs $(1.24 \mathrm{~mL})$ were diluted in $10.85 \mathrm{~mL}$ of borate buffer (buffer $\mathrm{A}, \mathrm{pH}$ 9.5, $9 \mathrm{mM}, \mathrm{I}=6 \mathrm{mM})$ or $\mathrm{PB}(\mathrm{pH} 7.4,2.7 \mathrm{mM}, \mathrm{I}=6 \mathrm{mM})$ to alter the $\mathrm{pH}$, followed by addition of freshly diluted anti-hFT dissolved in the same buffer $(372 \mu \mathrm{L}, 1 \mathrm{mg} / \mathrm{mL})$ for immobilization. After 1, 5, 10, 20, 30, 60, and $90 \mathrm{~min}$, the suspension (300 $\mu \mathrm{L})$ was removed and centrifuged $\left(15,000 \mathrm{rpm}, 4 \mathrm{~min} \times 2\right.$ or $10 \mathrm{~min} \times 1,25^{\circ} \mathrm{C}$; KR-20000; KUBOTA Co., Tokyo, Japan) to obtain the supernatant $(250 \mu \mathrm{L})$, which was used to quantify free antibodies with the Micro BCA assay. Alternatively, the construction and purification of SAP immunolatex for immunoreactivity measurements was carried out as previously described. SAP immunolatex separately prepared at $\mathrm{pH} 7.4$ and $\mathrm{pH} 9.5$ are referred to as $\operatorname{SAP}(7.4)$ and SAP(9.5), respectively.

After PEGylation, free anti-hFT content was checked to confirm released antibody after immunolatex formulation. To exclude the contribution from mPEG-N6, which also gave some color in the assay [27], PEGylated EDC-activated sMPs without antibodies were used as a control. This is a reasonable treatment because mPEG-N6 weakly colored the assay and the coupled mPEG-N6 was present at low concentations [27].

\subsubsection{Immunoreactivity measurements.}

We measured the immunoreactivity of purified SAP immunolatex with an automatic Biokit 
Quantex Analyser (BQA 5802-0149; Furuno Electric Co., Ltd., Japan) on the basis of the particle-enhanced immunoagglutination phenomenon. (Scheme 2) Briefly, a given amount of hFT (30 $\mu \mathrm{L}$, pH 7.0, containing $50 \mathrm{mM}$ Tris, $1 \mathrm{~g} / \mathrm{L} \mathrm{NaN}_{3}, 100 \mathrm{mM} \mathrm{NaCl}$, and $\left.10 \mathrm{~g} / \mathrm{L} \mathrm{BSA}\right)$ at room temperature was mixed with assay buffer at $8^{\circ} \mathrm{C}(190 \mu \mathrm{L}, \mathrm{PB}$ buffer, $\mathrm{pH} 7.4,10 \mathrm{mM}$, containing $1 \mathrm{~g} / \mathrm{L} \mathrm{BSA}$ and $\left.0.9 \mathrm{~g} / \mathrm{L} \mathrm{NaN}_{3}\right)$ in a cuvette at $37^{\circ} \mathrm{C}$. Then, SAP immunolatex (50 $\mu \mathrm{L}$, at $8^{\circ} \mathrm{C}$; sMPs concentration: $0.1 \% \mathrm{w} / \mathrm{v}$ ) was added in and mixed well immediately. The increment in the absorbance at $570 \mathrm{~nm}\left(\Delta \mathrm{Abs}_{570 \mathrm{~nm}}\right)$, which was generated from the rapid agglutination of particles specifically triggered by antibody-antigen recognition, was monitored immediately for $\sim 3$ min and defined as the immunolatex immunoreactivity.

\subsubsection{Circular dichroism (CD) and fluorescence measurements.}

After an original anti-hFT solution was diluted in buffers $(\mathrm{I}=6 \mathrm{mM})$ at different $\mathrm{pH}$ levels $(\mathrm{pH}=7.4,8.5$, and 9.5), circular dichroism (CD) and fluorescence spectra were monitored every 10 min for $1 \mathrm{~h}$. To avoid $\mathrm{pH}$ changes during the assay, the cell was sealed with parafilm. The CD spectra were recorded on a J-720W spectropolarimeter (Jasco, Japan), with a 1-mm quartz cell for far-ultraviolet radiation $(260$ to $200 \mathrm{~nm}$ ) and a $10-\mathrm{mm}$ quartz cell for near-ultraviolet radiation $(320$ to $260 \mathrm{~nm})$ in a temperature-controlled $\left(25^{\circ} \mathrm{C}\right)$ cuvette holder. The anti-hFT concentrations were 0.1 and $1 \mathrm{mg} / \mathrm{mL}$, respectively. The scan speed was 200 $\mathrm{nm} / \mathrm{min}$, which was the average of 10 scans for each measurement. The CD spectra were corrected for the buffer blank, and the mean residue ellipticities were calculated using a value 
of 110 for the mean residue weight. The fluorescence spectra for anti-hFT were recorded at a concentration of $0.5 \mathrm{mg} / \mathrm{mL}$ on an F-7000 fluorometer (Hitachi High-Technologies Co., Japan), with a $1-\mathrm{cm}$ quartz cell in a temperature-controlled $\left(25^{\circ} \mathrm{C}\right)$ cuvette holder. The excitation wavelength was $280 \mathrm{~nm}$, and the emission spectrum was between 290 to $450 \mathrm{~nm}$.

\subsubsection{Orientation assay of bound anti-hFT.}

The orientation assay of bound anti-hFT was performed on a PL-2500 spectrophotometer (Shimadzu, Japan). Briefly, a given amount of human serum containing $29 \mathrm{ng} / \mathrm{mL}$ of RF was poured into PB buffer (pH 7.4, $10 \mathrm{mM}$ ) to a final volume of $450 \mu \mathrm{L}$. Then, $50 \mu \mathrm{L}$ of purified SAP immunolatex (sMPs concentration of $0.1 \% \mathrm{w} / \mathrm{v}$ ) was added and mixed well, and the absorbance was immediately monitored at a wavelength of $550 \mathrm{~nm}$ for $5 \mathrm{~min}$ at room temperature. Because RF is an IgM antibody, which comprises multiple units specific for recognizing the Fc region of human and rabbit IgG [29,30], it may agglutinate the SAP immunolatex containing rabbit anti-hFT with the Fc region facing outward, which we term the "head-on" orientation shown in Scheme 3. The absorbance thus increased because of the assembled SAP particles. The higher the absorbance increment $\left(\Delta \mathrm{Abs}_{550 \mathrm{~nm}}\right)$ within a 5 min-period, the more "head-on" orientated bound anti-hFTs the SAP immunolatex has. In a parallel experiment, human serum without RF was used to confirm that RF specifically triggered SAP immunolatex agglutination.

2.2.6. Dynamic light scattering $(D L S)$ and electrophoretic mobility $\left(\mu_{e}\right)$ measurements. 
A Malvern Zetasizer Nano ZS instrument (He-Ne laser, 633 nm, Worcestershire, U.K.) was used for dynamic light scattering (DLS) and electrophoretic mobility $\left(\mu_{\mathrm{e}}\right)$ measurements at $25^{\circ} \mathrm{C}$. All test samples were measured after dilution in borate buffer $(\mathrm{pH} 8.0,10 \mathrm{mM}, \mathrm{I}=0.6$ $\mathrm{mM}$ ) to a final sMP concentration of approximately $2.5 \times 10^{-3} \% \mathrm{w} / \mathrm{v}$. Notably, the borate buffer's low ionic strength is a prerequisite for evaluating the variation in $\mu_{\mathrm{e}}$ values; otherwise, the surface charge will be thoroughly screened by ions. Each measurement was repeated at least 4 (DLS) or $3\left(\mu_{\mathrm{e}}\right)$ times, and the mean value was reported as the result.

\subsubsection{Statistics analysis.}

The results shown in this study are all expressed as average \pm S.D. $(n=3)$, except for the $\mathrm{CD}$ and fluorescence measurements. $\mathrm{P}$ values were calculated based on the t-test analysis.

\section{Results}

\subsection{Antibody immobilization at different $p H$ levels.}

To evaluate the effects of $\mathrm{pH}$ on antibody immobilization, anti-hFT was chemically bound to sMPs in buffers $(\mathrm{I}=0.6 \mathrm{mM}$ without salt $)$ at several $\mathrm{pH}$ levels. The amounts of both bound and unbound anti-hFT were then quantified. Figure 1a shows the pH-dependent amounts of free $\left(\Gamma_{\mathrm{f}}\right)$ and bound anti-hFT $\left(\Gamma_{\mathrm{b}}\right)$ in the system before PEGylation, as calculated with the Micro BCA assay. As the $\Gamma_{\mathrm{f}}$ increased, the $\Gamma_{\mathrm{b}}$ gradually decreased with increasing $\mathrm{pH}$. These results confirm that high $\mathrm{pH}$ values disfavor anti-hFT immobilization, in agreement with the results of previous reports, which show that the maximum amount of adsorbed proteins 
appeared at a $\mathrm{pH}$ value close to the pI $[21,22,25]$. After PEGylation to construct the SAP immunolatex, immunoreactivity measurements were performed at a range of antigen concentrations. Figure $1 \mathrm{~b}$ summarizes the SAP immunolatex immunoreactivity, which reflects the antigen recognition of immobilized anti-hFT. Interestingly, SAP immunolatex immunoreactivity increased with increasing $\mathrm{pH}$, contrary to the $\Gamma_{\mathrm{b}}$ decrease stated above. For example, the SAP immunoreactivity at $\mathrm{pH} 9.5$ is twice that at $\mathrm{pH} 7.4$ with an hFT concentration of $56 \mathrm{ng} / \mathrm{mL}$, as shown in the insert in Figure $1 \mathrm{~b}$.

\subsection{Circular dichroism (CD) and fluorescence measurements.}

Because proteins, such as antibodies, are susceptible to changes in environments, it is important to confirm structural changes during modification treatment. Both the anti-hFT CD and fluorescence spectra were monitored at 10-min intervals, following the dilution of the original anti-hFT into buffers at different $\mathrm{pH}$ levels, similar to the anti-hFT dilution treatment just before antibody immobilization. Figure 2 shows the anti-hFT far-ultraviolet and near-ultraviolet CD spectra, which were recorded immediately after anti-hFT dilution. These CD spectra showed no environment-induced changes even at the 60-min time point (Fig. S1 in Supporting Information). Anti-hFT fluorescence spectra did not vary, regardless of $\mathrm{pH}$ and time point (Fig. S2 in Supporting Information). The far- and near-ultraviolet CD are sensitive to variations in secondary and tertiary protein structures, respectively, and fluorescence intensity and maximum wavelength are also suitable indicators of changes in tertiary structure 
[31]. These results show that the anti-hFT structure did not change within 60 min at different pH levels.

\subsection{Effects of antibody-binding time.}

To gain an insight into the high anti-hFT antigen recognition at basic $\mathrm{pH}$, anti-hFT immobilization at a series of binding times was separately carried out at $\mathrm{pH} 7.4$ and 9.5. Figure 3 shows the $\Gamma_{\mathrm{b}}$ values both before and after PEGylation, which represents the amount of surface antibody after the immobilization reaction (Fig. 3a) and after PEGylation (Fig. 3b). Before PEGylation (Fig. 3a), almost all anti-hFT was bound to sMPs at $\mathrm{pH} 7.4$, which was observed even after $1 \mathrm{~min}$ (theoretical $\Gamma_{\mathrm{b}}$ for complete binding $=29.9 \mu \mathrm{g} / \mathrm{mL}$ ), resulting in a nearly constant $\Gamma_{\mathrm{b}}$. On the contrary, binding at $\mathrm{pH} 9.5$ proceeded slowly, and free anti-hFT remained after $90 \mathrm{~min}$. These data clearly suggest that the anti-hFT adsorption rate is extremely high at $\mathrm{pH} 7.4$, but low at $\mathrm{pH}$ 9.5. After PEGylation (Fig. 3b, theoretical $\Gamma_{\mathrm{b}}$ for complete binding $=19.9 \mu \mathrm{g} / \mathrm{mL}$ ), large amounts of pre-bound anti-hFT were unexpectedly liberated from sMPs, especially those with short binding times ( $<20 \mathrm{~min})$. Consequently, $\Gamma_{\mathrm{b}}$ displayed a similar time-dependency at both $\mathrm{pH} 7.4$ and 9.5. Considering the experimental error (see Materials and methods), the slight difference in $\Gamma_{\mathrm{b}}$ between $\operatorname{SAP}(7.4)$ and $\operatorname{SAP}(9.5)$ was negligible. Release of antibody was triggered by PEGylation because anti-hFT release from sMPs was not observed following addition of PB (pH 7.4, 8 mM) (Fig. S3 in Supporting Information). Probably, a large amount of mPEG-N6 competitively replaced weakly adsorbed 
antibodies before their covalent linkage formation, especially the case with short binding time. Figure 4 shows the antigen recognition to bound anti-hFT as a function of the antibody-binding time. The recognition of SAP(9.5) was much higher than that of SAP(7.4), particularly at short binding times and high antigen concentrations. Although the SAP(7.4) and $\operatorname{SAP}(9.5) \Gamma_{\mathrm{b}}$ values increased with time up to 90 min (Figure $3 \mathrm{~b}$ ), we did not observed a significant increase in antigen recognition, especially for SAP prepared with a long antibody-immobilization time. Excessive antibody immobilization [32] has been shown to decrease antigen recognition because of steric restriction. However, in our experiments, the reactivity increased at higher concentrations of immobilized antibody (Fig. S4 in Supporting Information). The different antigen affinities clearly indicate that the amount of immobilization antibody is not responsible for the high $\operatorname{SAP}(9.5)$ immunoreactivity.

\subsection{Orientation assay of immobilized anti-hFT.}

As described in the Materials and methods, RF IgM may specifically recognize the anti-hFT Fc region. Therefore, the high $\Delta \mathrm{Abs}_{550 \mathrm{~nm}}$ value denotes that the SAP immunolatex contains more "head-on" orientated anti-hFT molecules (Scheme 3). Figure 5a shows the $\mathrm{SAP}(7.4)$ and $\mathrm{SAP}(9.5) \Delta \mathrm{Abs}_{550 \mathrm{~nm}}$ values, both with an antibody-immobilization time of 20 min, as a function of the volume of RF-containing human serum. Although the 2 values increased with an increase in the RF amount, the $\Delta \mathrm{Abs}_{550 \mathrm{~nm}}$ value was much higher for $\mathrm{SAP}(7.4)$ than for $\mathrm{SAP}(9.5)$ under the same conditions. The SAP immunolatex $\Delta \mathrm{Abs}_{550 \mathrm{~nm}}$ 
value obtained with human serum without RF is zero, regardless of the amount of human serum used. The results confirm that RF specifically agglutinated the SAP immunolatex and that SAP(7.4) contained more nonreactive "head-on" orientated anti-hFT than did SAP(9.5). Notably, SAP(7.4) and SAP(9.5) used in this experiment had the same $\Gamma_{b}$ (Fig. 3b). Thus, their different $\Delta \mathrm{Abs}_{550 \mathrm{~nm}}$ values reflect different orientation states of bound anti-hFT. The correlation between the $\Delta \mathrm{Abs}_{550 \mathrm{~nm}}$ value of SAP immunolatex and the antibody-binding time was then estimated using the same amount of RF ( $3 \mu \mathrm{L}$ of RF-containing human serum), and the resultant correlation profiles are summarized in Figure $5 \mathrm{~b}$. Unike the $\Delta \mathrm{Abs}_{550 \mathrm{~nm}}$ of SAP(7.4), which increased immediately after antibody immobilization, the SAP(9.5) $\Delta \mathrm{Abs}_{550 \mathrm{~nm}}$ increased only at the later time points in the antibody-immobilization experiment, suggesting that a long antibody-binding time tends to cause orientation changes under alkaline conditions. Figure $5 \mathrm{c}$ provides the $\Delta \mathrm{Abs}_{550 \mathrm{~mm}} \Gamma_{\mathrm{b}}$-dependency calculated from the results shown in Figures $5 b$ and $3 b$, for which linear and concave upward $\Gamma_{b}$-dependency curves were separately obtained for $\operatorname{SAP}(7.4)$ and $\operatorname{SAP}(9.5)$. On the basis of this data, we suggest that the antibody that immobilized under alkaline conditions was probably highly oriented. Its orientation, however, gradually changed during the immobilization process under the same conditions.

\subsection{Electrophoretic mobility $\left(\mu_{e}\right)$ measurements.}

We tested the SAP immunolatex electrophoretic mobility $\left(\mu_{\mathrm{e}}\right)$ to obtain further information 
about the antibody's surface, and we observed some intriguing phenomena. The SAP immunolatex $\mu_{\mathrm{e}}$ is presented as a function of the antibody-binding time (Fig. 6a) and of its $\Gamma_{\mathrm{b}}$ (Fig. 6b). The $\mu_{\mathrm{e}}$ value of $\operatorname{SAP}(9.5)$ was much higher than that of $\operatorname{SAP}(7.4)$ at a binding time of 1 min. However, this value continuously decreased over time in the case of $\operatorname{SAP}(9.5)$, while we did not observed a change in the $\mu_{\mathrm{e}}$ value of $\operatorname{SAP}(7.4)$. Finally, the $\mu_{\mathrm{e}}$ values for $\operatorname{SAP}(7.4)$ and $\operatorname{SAP}(9.5)$ became equal. A rapid decrease in the $\mu_{\mathrm{e}}$ value of $\mathrm{SAP}(9.5)$ occurred at $\Gamma_{\mathrm{b}}>10 \mu \mathrm{g} / \mathrm{mL}$, accompanied with an invariable $\mu_{\mathrm{e}}$ value of $\operatorname{SAP}(7.4)$. Proteins tend to change their conformation to spread on the surface after binding with each other to enhance their interaction [14], and adsorption retardation, such as that at $\mathrm{pH} 9.5$, further promoted this tendecy [24]. Because of the conformational changes in the antibody after a prolonged antibody-immobilization phase, the remaining surface might be significantly reduced, thereby restricting subsequent PEGylation and resulting in a decreased $\mu_{\mathrm{e}}$ value. We assumed that the relatively low PEGylation effectiveness at neutral $\mathrm{pH}$, in comparison with that at basic $\mathrm{pH}$ [33], accounts for the constant low $\operatorname{SAP}(7.4) \mu_{\mathrm{e}}$ values. Otherwise, a dense PEG layer might efficiently shield the surface charge to give a high $\mu_{\mathrm{e}}$ value. Actually, we found that the $\mu_{\mathrm{e}}$ values of PEGylated sMPs (sMP/PEG complex) under the same conditions without antibody were -2.4 (at $\mathrm{pH} 9.5$ ) and $-2.7($ at $\mathrm{pH} 7.4) \mu \mathrm{m} \mathrm{cm} / \mathrm{Vs}$, and both values were almost constant within $60 \mathrm{~min}$. The PEGylation results will be reported elsewhere.

\section{Discussion}


As described in the Introduction, the physisorption properties of anti-hFT might play an important role in its chemical immobilization reaction. On the basis of the sMP surface and anti-hFT (pI = 6 7) characteristics, the hydrophobic effect and the electrostatic interactions, in particular, are crucial for efficient anti-hFT adsorption. Compelling evidence was obtained from the anti-hFT immobilization at $\mathrm{pH} 9.5$ in the presence of salt $(3-9 \mathrm{mM} \mathrm{NaCl}) . \Gamma_{\mathrm{b}}$ significantly increased as salt concentration increased, whereas no variation was observed in $\Gamma_{\mathrm{b}}$ at $\mathrm{pH} 7.4$ under the same conditions (Table S1 in Supporting Information). Salt decreased the electrostatic repulsive force between anionic anti-hFT and the negatively charged sMP surface at high $\mathrm{pH}$, confirming that the electrostatic repulsive force retards the access of the antibody to the sMP surface under alkaline conditions.

Generally, there are three possibilities that may account for increased immunoreactivity: (1) a net increase in antibody's antigen recognition before immobilization, (2) increased antibody immobilization (or antibody-surface concentration), and (3) changes in the orientation of bound antibodies $[16,17,26]$. With regards to an increase in binding recognition, we evaluated structural variations in anti-hFT in terms of time-dependent circular dichroism (CD) and fluorescence spectra at different $\mathrm{pH}$ levels. We did not observe any variation in the anti-hFT structure within 60 min. (Fig. 2) Since the anti-hFT dilution was usually performed less than 5 min before immobilization, the structure of anti-hFT should not have changed significantly. Moreover, the whole SAP immunolatex preparation, including both antibody immobilization 
and PEGylation, took place over the course of an hour. Therefore, increased antigen recognition of anti-hFT prior to immobilization seems unlikely. An increased amount of surface antibody is also unlikely because $\operatorname{SAP}(7.4)$ and $\operatorname{SAP}(9.5)$ had similar $\Gamma_{\mathrm{b}}$ values despite the difference in the antibody binding time (Fig. 3b). To ascertain whether changes in orientation could account for our results, the orientation of bound anti-hFT was directly analyzed with human serum containing Fc-region-recognizable RF. As shown in Figure 5b, the initial SAP (9.5) $\Delta \mathrm{Abs}_{550 \mathrm{~nm}}$ value was much lower than that of $\operatorname{SAP}(7.4)\left(\Gamma_{\mathrm{b}}<10 \mu \mathrm{g} / \mathrm{mL}\right)$, suggesting the presence of differently oriented immobilized antibodies at $\mathrm{pH} 7.4$ and $\mathrm{pH} 9.5$. As the amount of immobilized antibody increased, however, more SAP(9.5)-derived anti-hFT molecules with Fc regions facing outward gradually appeared on the surface (Fig. 5c), confirming that the increased amount of pre-bound antibody changed the orientation of post-bound one. Moreover, the $\mu_{\mathrm{e}}$ values of $\mathrm{SAP}(9.5)$ further supported our results. The $\Gamma_{\mathrm{b}}$-dependent $\mu_{\mathrm{e}}$ value (Fig. 6b) and $\Delta \mathrm{Abs}_{550 \mathrm{~nm}}$ (Fig. 5b) of SAP(9.5) were mostly constant at $\Gamma_{\mathrm{b}}<10 \mu \mathrm{g} / \mathrm{mL}$ before continuous variation.

On the basis of these results, it is plausible that the differences in immunoreactivity between SAP(7.4) and SAP(9.5) originated from different orientations of bound anti-hFT. At $\mathrm{pH}$ 7.4, almost neutral anti-hFT might strongly prefer hydrophobic attractions with sMPs, resulting in an extremely rapid or instant adsorption process and random orientation (Scheme 4a). The enhanced electrostatic repulsive force at $\mathrm{pH} 9.5$ compensated for the hydrophobic 
interaction, retarding anti-hFT adsorption onto the negatively charged surface. In principle, IgG adsorption preferentially occurs with its Fc region on the hydrophobic surface, owing to its hydrophobic characteristics and/or the structural instability over the IgG antigen-binding region $\left(\mathrm{F}\left(\mathrm{ab}^{\prime}\right)_{2}\right)$ [24]. Additionally, the anti-hFT $\mathrm{F}\left(\mathrm{ab}^{\prime}\right)_{2}$ region $(\mathrm{pI}=\sim 5.6)$ [34] should be negatively charged at high $\mathrm{pH}$ and electrostatically repelled from sMPs. Thus, retardation of anti-hFT adsorption may provide time for anti-hFT orientation changes, such as the change to the "end-on" orientation (Fc region close to the surface as shown in Scheme 3), which favor antibody-antigen recognition (Scheme $4 b$ ). We named this the "soft landing" mechanism. It should be emphasized here that subsequent PEGylation played a key role in maintaining the immobilized antibody's orientation, as reported previously [13]. However, this situation changed as the amount of surface antibody increased, probably because of weakened repulsive forces, leading to randomly oriented bound antibody similar to that found at $\mathrm{pH}$ 7.4. In addition, the pre-bond antibodies might also change orientation and/or conformation at longer antibody-binding times before PEGylation, thus losing antigen recognition [13]. Consequently, $\operatorname{SAP}(9.5)$ showed higher antigen recognition than $\operatorname{SAP}(7.4)$, but both values remained constant at later time points. (Fig. 4)

\section{Conclusion}

By physically manipulating anti-hFT orientation during chemical immobilization, we successfully formed a high-performance SAP immunolatex. The detailed study on anti-hFT 
immobilization with respect to the extent of immobilization, antigen recognition, and anti-hFT orientation, supports the idea of a rapid blocking effect of PEGylation following short-term antibody immobilization. This effect benefited from the combination of physical adsorption and covalent binding of antibodies. Physical adsorption may have resulted in facile orientation changes and covalent binding may have anchored antibodies robustly, unlike traditional time-consuming antibody immobilization protocols. Moreover, the consistency of the $\Gamma_{\mathrm{b}}$-dependent variations in antibody orientation and the SAP immunolatex $\mu_{\mathrm{e}}$ values implies that electrophoretic mobility measurements probably provide a convenient indirect approach to investigate antibody orientation, which relies on the inherent structural nature of the antibody/PEG hybrid layer on sMPs. It should be mentioned here that the proper $\mathrm{pH}$ level for obtaining proper antibody orientation may be adjusted down to close to antibody's PI by using highly charged sMPs, if antibodies are not stable enough at high $\mathrm{pH}$ level during the binding process. Thus, although more experiments are still required, the "soft landing" mechanism proposed in this study seems a general rule for antibody binding and will attract more attention as a useful tool in the near future.

Acknowledgments: This research was supported by Biokit S.A. (Barcelona, Spain) and a Grant-in-Aid for Scientific Research on Innovative Areas (Soft Interface \# 20106011) from the Ministry of Education, Culture, Sports, Science and Technology (MEXT) of Japan.

\section{Appendix A. Supplementary data}


Supplementary data associated with this article can be found, in the online version, at 


\section{References}

[1] S. K. Mwilu, A. O. Aluoch, S. Miller, P. Wong, O. A. Sadik, Anal. Chem. 81 (2009) 7561.

[2] M. B. Sosellner, K. A. Dickson, B. L. Nilsson, R. T. Raines, J. Am. Chem. Soc. 125 (2003) 11790.

[3] T. Cha, A. Guo, X. Zhu, Proteomics 5 (2005) 416.

[4] A. Watzke, M. Kohn, M. Gutierrez-Rodriguez, R. Wacker, H. Schroder, R. Breinbauer, J. Kuhlmann, K. Alexandrov, C. M. Niemeyer, R. S. Goody, H. Waldmann, Angew. Chem. Int. Ed. 45 (2006) 1408.

[5] Z. Suo, X. Yang, R. Avci, M. Deliorman, P. Rugheimer, D. W. Pascual, Y. Idzerda, Anal. Chem. 81(2009) 7571.

[6] H. Chon, S. Lee, S. W. Son, C. H. Oh, J. Choo, Anal. Chem. 81(2009) 3029.

[7] Y. Nagasaki, H. Kobayashi, Y. Katsytana, T. Jomura, T. Sakura, J. Colloid Interface Sci. 309 (2007) 524

[8] T. Tanaka, T. Matsunaga, Anal. Chem. 72 (2000) 3518.

[9] J. M. Peula, R. Hidalgo-Alvarez, F. J. de las Nieves, J. Colloid Interface Sci. 201(1998) 132.

[10] P. Peluso, D. S. Wilson, D. Do, H. Tran, M. Venkatasubbaiah, D. Quincy, B. Heidecker, K. Poindexter, N. Tolani, M. Phelan, K. Witte, L. S. Jung, P. Wagner, S. Nock, Anal. Biochem. 312 (2003) 113 
[11] J. W. Chung, J. M. Park, R. Bernhardt, J. C. Pyun, J. Biotechnol. 126 (2006) 325.

[12] A. A. Karyakin, G. V. Presnova, M. Y. Rubtsova, A. M. Egorov, Anal. Chem. 72 (2000) 3805.

[13] K. Yoshimoto, M. Nishio, Y. Nagasaki, J. Am. Chem. Soc. 132 (2010) 7982.

[14] P. Lin, S. Chen, K. Wang, M. Chem, A. K. Adak J. Hwu, Y. Chen, C. Lin, Anal. Chem. $81(2009) 8774$.

[15] J. D. Conradie, M. Govender, L. Visser, J. Immunol. Methods 59 (1983) 289.

[16] I. N. Chang, J. N. Lin, J. D. Andrade, J. N. Herron, J. Colloid Interface Sci. 174 (1995) 10.

[17] R. van-Erp, Y. E. M. Linders, A. P. G. van-Sommeren, T. C. Gribnau, J. Immunol. Methods 152 (1992) 191.

[18] Y. Yuan, H. He, L. J. Lee, Biotechnol. Bioeng. 102 (2009) 891.

[19] Y. Jung. J. M. Lee, H. Jung, B. H. Chung, Anal. Chem. 79 (2007) 6534.

[20] H. Shen, J. Watanantibodye, M. Akashi, Anal. Chem. 81 (2009) 6923.

[21] J. L. Ortega-Vinuesa, M. J. Galvez-Ruiz, R. Hidalgo-Alvarez, Langmuir 12 (1996) 3211 .

[22] J. Buijs, J. W. T. Lichtenbelt, W. Norde, J. Lyklema, Colloids Surf. B 5 (1995) 11.

[23] J. Zhou, H. Tsao, Y. Sheng, S. Jiang, J. Chem. Phys. 121 (2004) 1050.

[24] J. Buijs, W. Norde, Langmuir 12 (1996) 1605. 
[25] S. Chen, L. Liu, J. Zhou, S. Jiang, Langmuir 19 (2003) 2859.

[26] J. N. Lin, J. D. Andrade, I. N. Chang, J. Immonol. Methods 125 (1989) 67.

[27] X. Yuan, K. Yoshimoto, Y. Nagasaki, Anal. Chem. 81 (2009) 1549.

[28] X. Yuan, D. Fabregat, K. Yoshimoto, Y. Nagasaki, Anal. Chem. 81 (2009) 10097.

[29] I. Giaever, C. R. Keese, R. I. Rynes, Clin. Chem. 30 (1984) 880.

[30] Y. Hashimoto, T. Ikenaga, Y. Tanigawa, T. Ueda, I. Ezaki, T. Imoto, Biol. Pharm. Bull. 23 (2000) 941.

[31] Y. Tsybovsky, D. V. Shubenok, Z. I. Kravchuk, S. P. Martsev, Protein Eng. Des. Sel 20 (2007) 481 .

[32] J. Buijs, D. D. White, W. Norde, Colloids Surf. B 8 (1997) 239.

[33] Information from Biokit S.A.

[34] N. Nakajima, Y. Ikada, Bioconjugate Chem. 6 (1995) 123. 


\section{Figure Captions}

Scheme 1. (a) Chemical structure of mPEG-N6 and (b) SAP immunolatex construction.

Scheme 2. Schematic representation of the immunoreactivity assay used in this study.

Scheme 3. Schematic illustration of the specific interaction between rheumatoid factor (RF) and the Fc region of bound anti-hFT.

Scheme 4. Proposed mechanism of anti-hFT immobilization at (a) pH7.4 and (b) pH9.5.

Figure 1. (a) $\mathrm{pH}$-dependent antibody amount of immobilized anti-hFT $\left(\Gamma_{\mathrm{b}}, \mathbf{0}\right)$ and free anti-hFT $\left(\Gamma_{\mathrm{f}}, \mathrm{O}\right)$ in the system before PEGylation measured by Micro BCA assay. (b) Immunoreactivity of SAP immunolatex as a function of hFT concentration (left) and $\mathrm{pH}$-dependent immunoreactivity of SAP immunolatex measured at $\mathrm{hFT}=56 \mathrm{ng} / \mathrm{mL}$ (right). The SAP immunolatex was prepared by immobilizing anti-hFT at $\mathrm{pH} 7.4(\diamond), \mathrm{pH} 8(\triangle)$, pH8.5 ( $\square)$, pH9 (O), and pH9.5 $(\times)$, respectively. * and \# : $\mathrm{P}<0.001$ separately vs. that at pH7.4; $\times$ : $\mathrm{P}<0.05$ vs. that at $\mathrm{pH} 8.0 ;+\mathrm{P}<0.05$ vs. that at $\mathrm{pH} 8.5$ based on t-test analysis $(\mathrm{n}=$ 3).

Figure 2. (a) Far-ultraviolet and (b) near-ultraviolet CD spectra recorded immediately (holding time $\mathrm{t}=0$ ) after dilution of anti-hFT in buffers at different $\mathrm{pH}$ levels. Solid line $(-)$, dotted line (...), and broken line (----) represent the spectra of anti-hFT in a buffer $(\mathrm{I}=6$ $\mathrm{mM})$ of $\mathrm{pH}=7.4,8.5$, and 9.5, respectively.

Figure 3. (a) Time-dependent anti-hFT quantities of sensitized sMPs before PEGylation, and its fraction of the total $\left(\Gamma_{\mathrm{b}} /\left(\Gamma_{\mathrm{b}}+\Gamma_{\mathrm{f}}\right)\right)$, as antibody binding was performed at pH7.4 $(\triangle)$ and pH9.5 (O), respectively. (b) Time-dependent anti-hFT quantities and its fraction of the total $\left(\Gamma_{\mathrm{b}} /\left(\Gamma_{\mathrm{b}}+\Gamma_{\mathrm{f}}\right)\right)$ after the construction of $\operatorname{SAP}(7.4)(\triangle)$ and $\operatorname{SAP}(9.5)(\bigcirc)$, respectively. $(\mathrm{n}=3)$

Figure 4. Correlation between anti-hFT binding time and $\operatorname{SAP}(7.4)$ (a) and $\operatorname{SAP}(9.5)$ (b) immunoreactivity, respectively. Immunoreactivity measurements were performed at hFT 
concentrations of 11 ( $\triangle$ and $\mathbf{\Delta}), 22(\diamond$ and $\diamond)$, and $56 \mathrm{ng} / \mathrm{mL}\left(\bigcirc\right.$ and $\bullet$ ), respectively. $*_{1}$, $\#_{1}$, and $+_{1}: \mathrm{P}<0.01$ separately vs. that of $\operatorname{SAP}(7.4)$ with antibody binding time $=1 \mathrm{~min} ; *_{2}, \#_{2}$, and $+_{2}: \mathrm{P}<0.05$ separately vs. that of $\operatorname{SAP}(9.5)$ with antibody binding time $=1 \mathrm{~min}$, both based on t-test analysis $(n=3)$.

Figure 5. (a) Variations in the $\triangle \mathrm{Abs}_{550 \mathrm{~nm}}$ value of SAP immunolatex (anti-hFT binding time $=$ 20 min) with human serum containing RF (open symbol) or not (close symbol); (b) Antibody-binding time dependent variations in SAP immunolatex $\Delta \mathrm{Abs}_{550 \mathrm{~nm}}$; (c) $\Gamma_{\mathrm{b}}$-dependent $\Delta \mathrm{Abs}_{550 \mathrm{~nm}}$ of SAP immunolatex. $\operatorname{SAP}(9.5)(\bigcirc$ and $\mathbf{O}) ; \operatorname{SAP}(7.4)(\triangle$ and $\boldsymbol{\Delta}) .(\mathrm{n}=3)$

Figure 6. (a) Antibody-binding time dependent variations in electrophoretic mobility $\left(\mu_{\mathrm{e}}\right)$ of SAP immunolatex. (b) $\Gamma_{\mathrm{b}}$-dependent SAP immunolatex $\mu_{\mathrm{e}} . \operatorname{SAP}(7.4)(\triangle) ; \operatorname{SAP}(9.5)(\bigcirc) .(\mathrm{n}=$ 3) 
a

MPEG-N6<smiles>COCCOCCN(C)CCCN[TlH]</smiles>

\section{b Immunolatex construction}

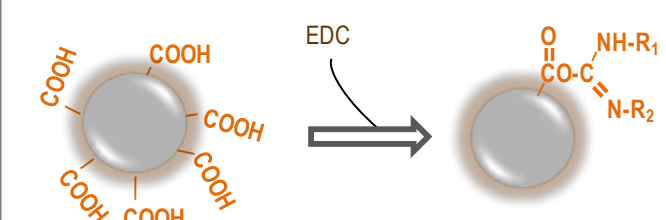

$\mathrm{COOH}$

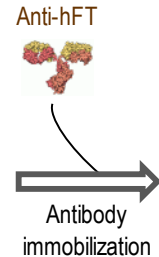

immobilization
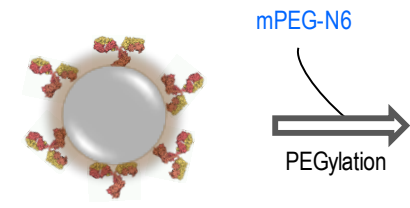

Sensitized SMPs

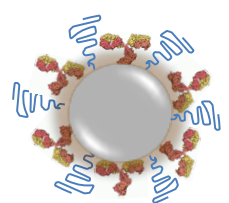

SAP immunolatex

Scheme 1 


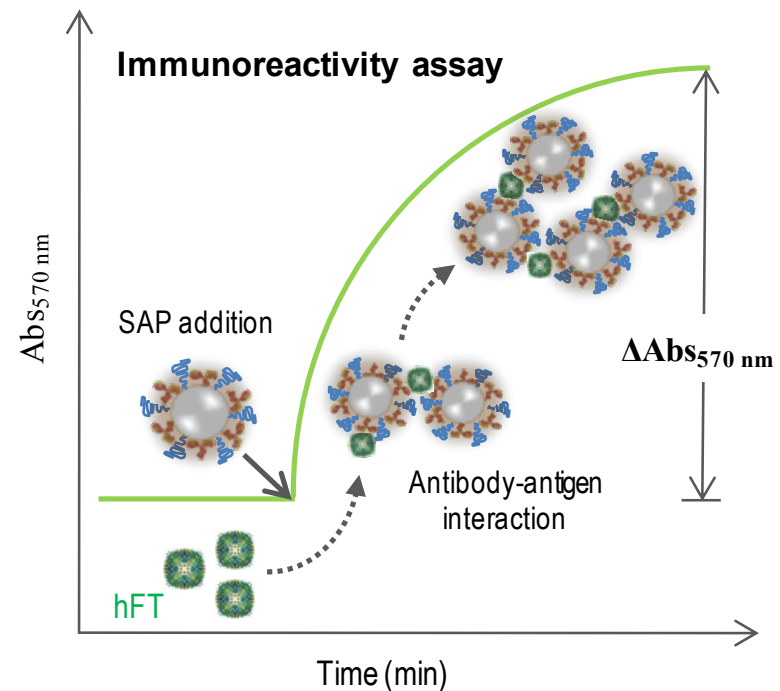

Scheme 2 


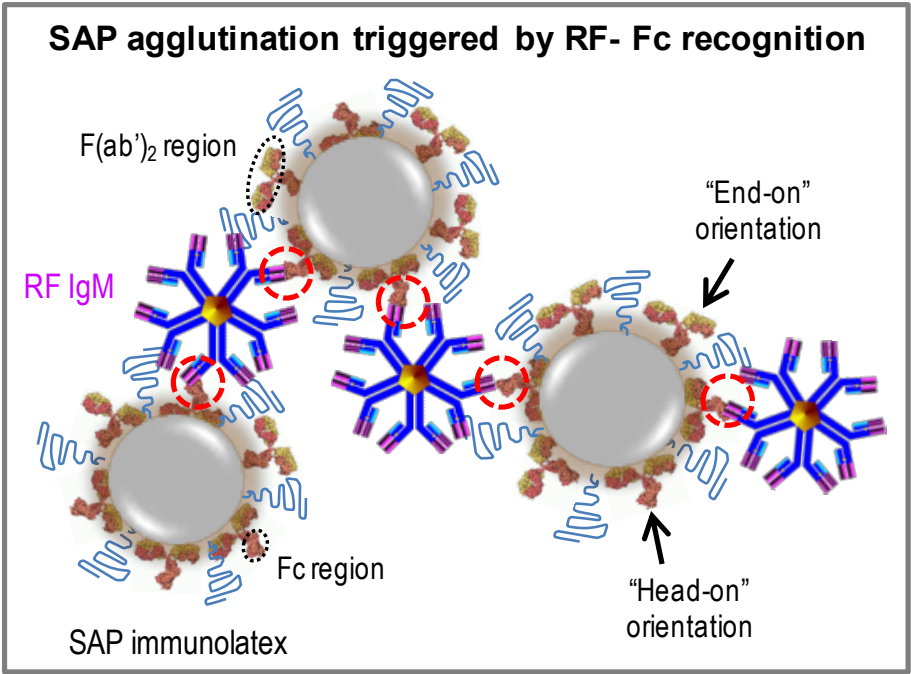

Scheme 3 


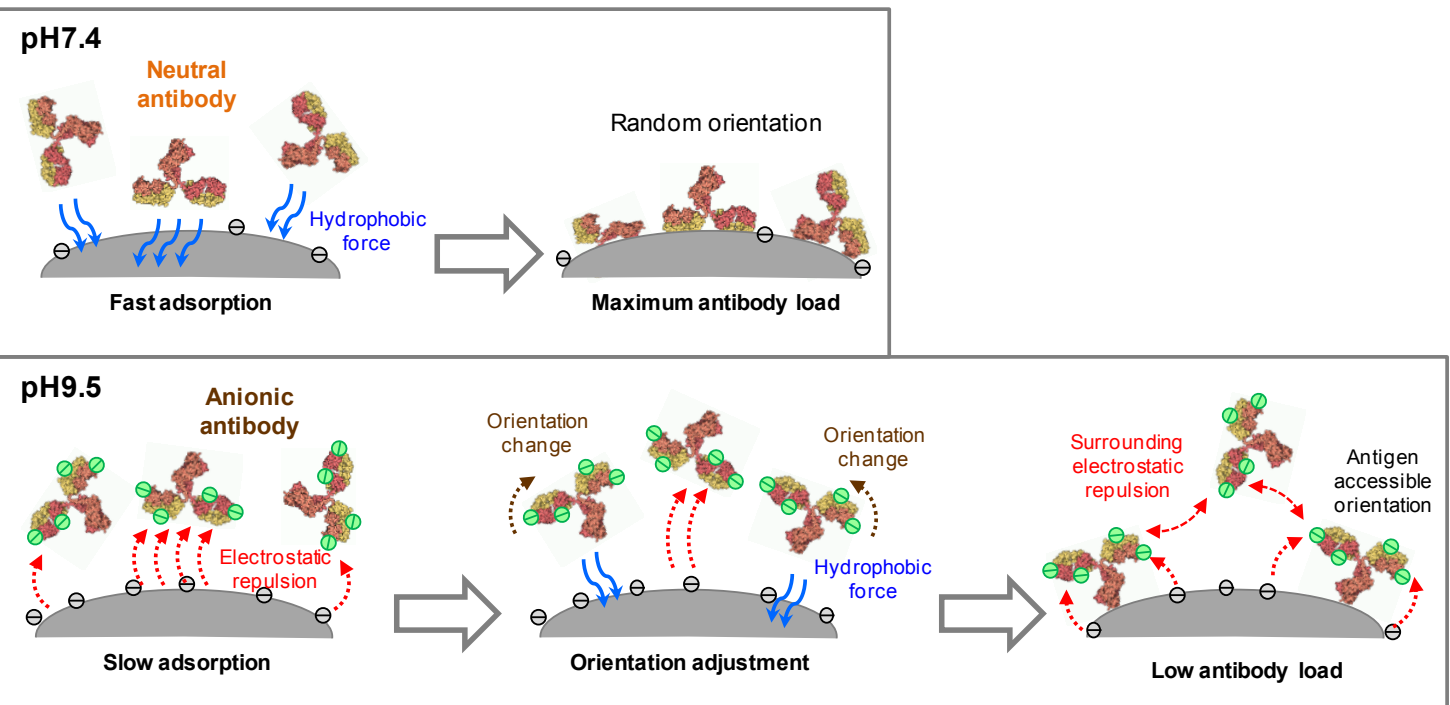

Scheme 4 

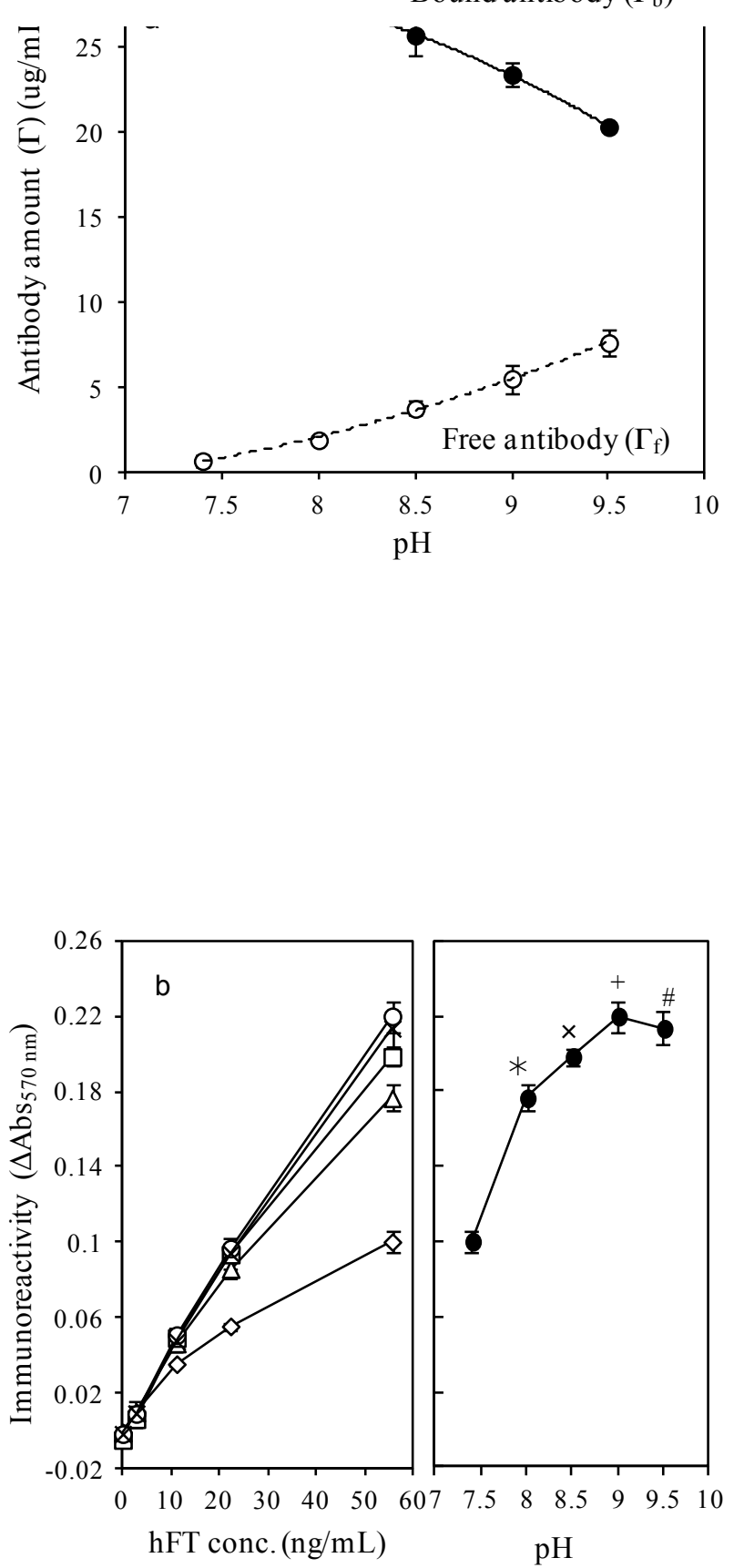

Figure 1 

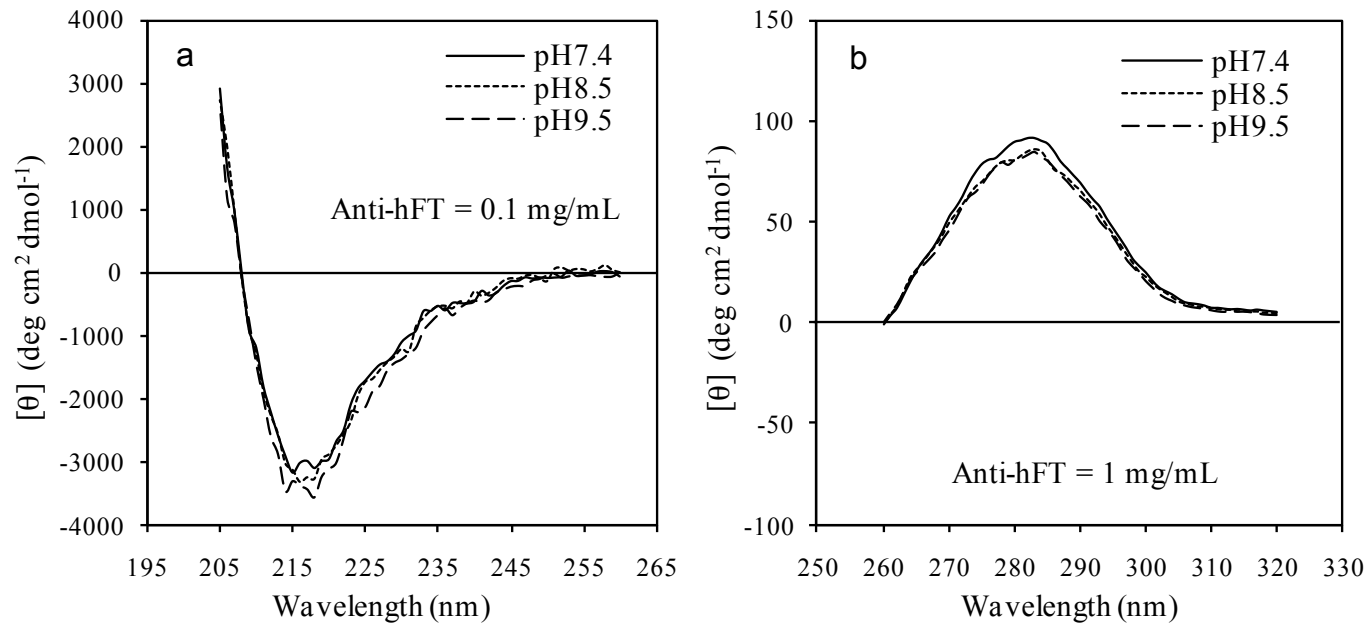

Figure 2 

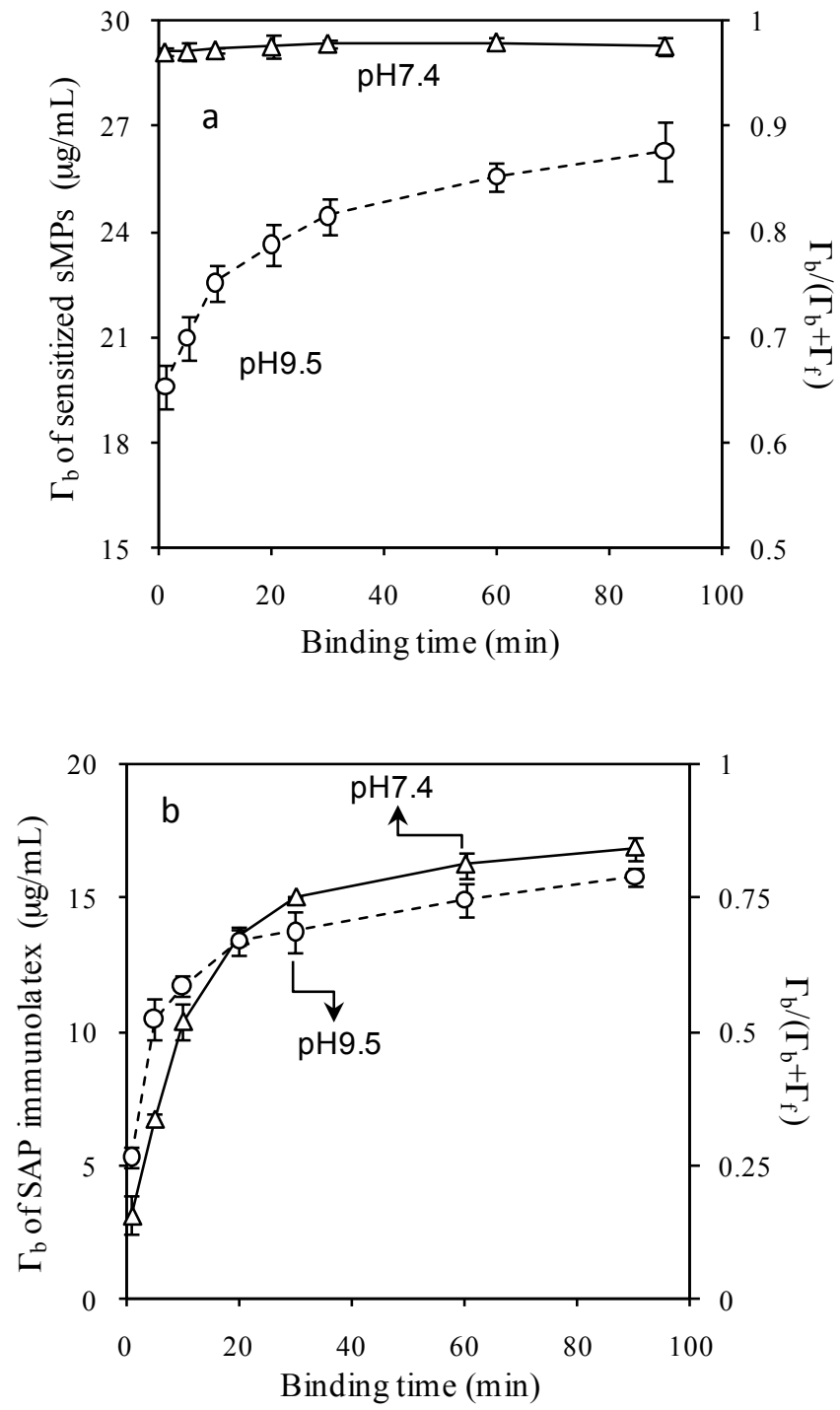

Figure 3 

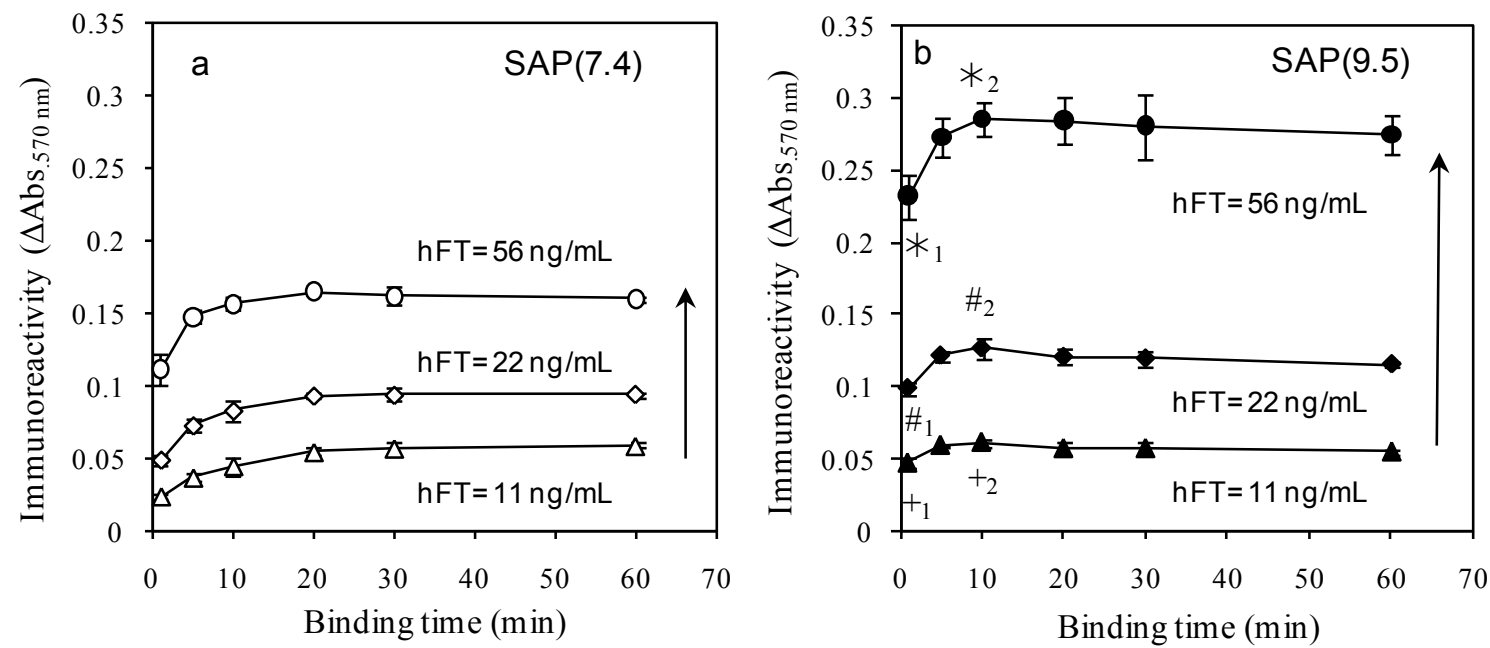

Figure 4 

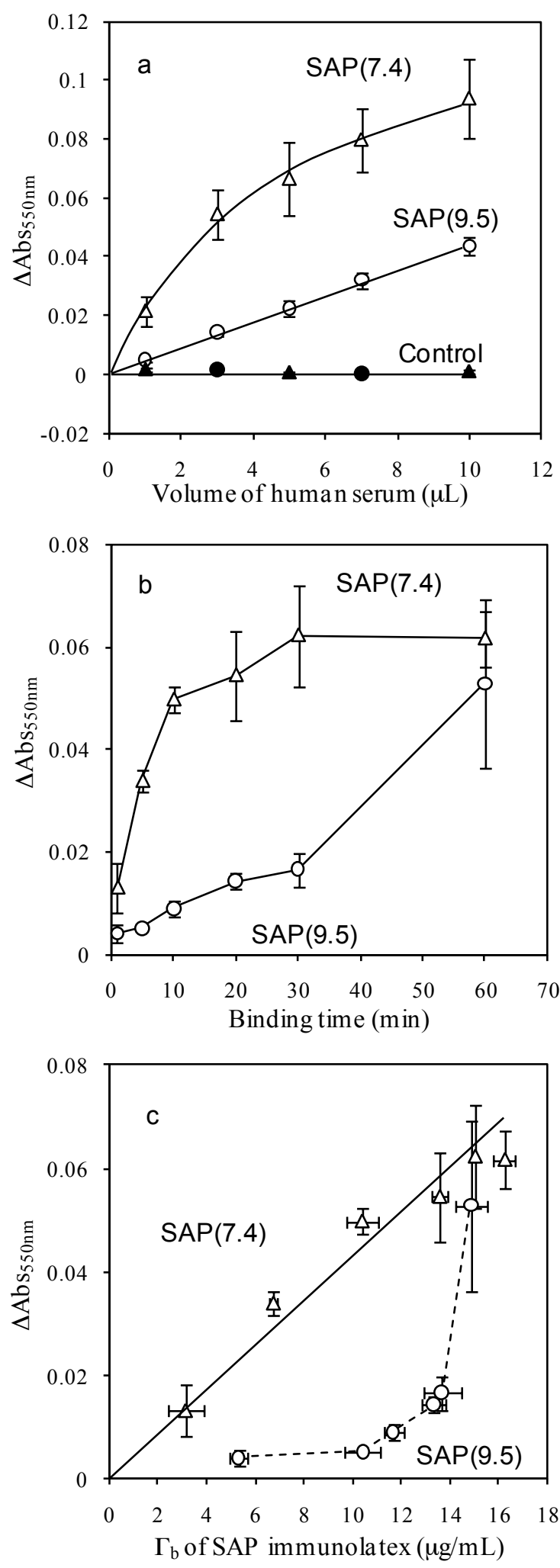

Figure 5 

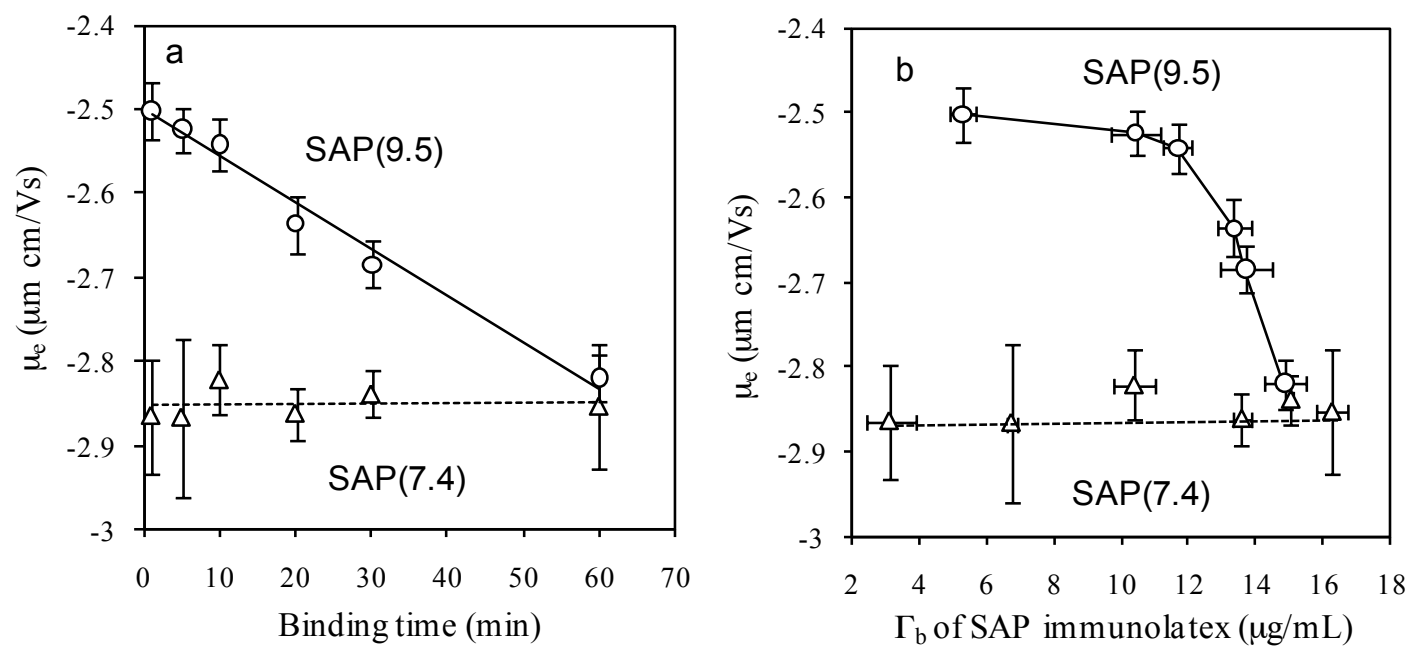

Figure 6 